\title{
Chemical Composition of Essential Oils of Released Black Cumin Varieties Grown in Ethiopia
}

\author{
Sileshi Abera, Biruk Hirko \\ Ethiopian Institute of Agricultural research, Tepi Agricultural Research Center \\ P.O.Box 34, Tepi, Ethiopia
}

\begin{abstract}
In Ethiopia black cumin seed and oil are used in folk medicine, as a bread flavoring and as a spices. The essential oil of black cumin seeds were extracted by hydro-distillation with Clevenger apparatus and the constituents of the three varieties were identified using GC/MS. The essential oil content of black cumin seeds were $0.80 \%, 0.4 \%$ and $0.6 \%$ for Eden, Dershy and Silengo respectively. The GC/MS results showed that seventy five, sixty six and sixty constituents were identified which constituting 99.98\%, 99.27\% and 99.17\% in Eden, Dershy and Silengo. The main constituents of Ethiopian black cumin verities seeds essential oil as detected by GC/MS ware p-cymene $(45.85-44.31 \%), \alpha$-thujen $(17.30-12.57 \%)$, trans-4-methoxy thujane (8.86 - 7.39\%), 9,12-Octadecanoic acid, 9Z,Z)- $(6.04 \%-0.07 \%), \beta$-pinene $(4.08-3.04 \%), \alpha$ - pinene $(3.94-2.68 \%)$, gamma-terpinene $(3.83-2.50 \%)$, thymoquinone $(3.53-2.13 \%), \alpha$-terpinene $(3.00-0.00 \%)$, D-limonene $(2.29-2.08 \%)$, phenol-2-methyl-5-(1methylethyl) (2.56-1.52\%), cis-vaccenic acid (2.46-0.00\%), longifolene (1.95-1.83\%), the results confirms that main compounds are similar and variation in content to each varieties. The Ethiopian black cumin essential oil contain the required major secondary metabolite for pharmacological and other application.
\end{abstract}

Keywords: Black cumin, Ethiopia, Essential oil, p-cymene,spices

DOI: $10.7176 / \mathrm{CMR} / 12-2-02$

Publication date: February $29^{\text {th }} 2020$

\section{Introduction}

A Spice is a natural compound that is extracted from the seed, fruits, flowers or trunks of several plants are add to food in order to provide taste smell or flavor. Spices are a diverse group of wide variety of staple dietary additives consumed all over the world. The spice is a culinary term not a botanical category it does not refer to a specific kind of plant or plant part [1].

Each spice has a unique aroma and flavor which derive from compounds known as phytochemical or secondary metabolite. These chemicals evolved in plant to protect them against herbivorous insect, vertebrates, fungi pathogens and parasites [2-4]

Black cumin or Nigella sativa L (N. sativa) belongs to the family Ranunculaceae and is widely distributed and cultivated in Mediterranean countries, middle Europe and western Asia. The ancient Egyptians, Greeks and Romans were already aware of the therapeutic properties of $N$. sativa, the essential oil and seeds of which are still used in folk medicine, as a bread or cheese flavoring and as a spice in various kinds of meals [5].

The seeds have been reported to contain mainly fixed oils, proteins, alkaloids, saponins, and essential oil that was previously characterized by a higher percentage of monoterpenes, the main constituents being thymoquinone and $p$-cymene [6].

Black seed, the seed of Nigella sativa L. (Ranunculaceae), has been employed for thousands of years as a spice, food preservative and curative remedy for numerous disorders [7]. The historical tradition of black seed use in medicine is substantial. N. sativa is known to have beneficial effects on a wide range of diseases, antiasthmatic, antitumor, antiviral, antibacterial, anti-inflammatory, antimalarial, antihypertensive, antidiabetic, [8-12]

Thymoquinone, the main constituent of the essential oil of $N$. sativa seeds, was capable to also exert beneficial effects on acute gastric ulcer [13]. In addition, thymoquinone and its reduced product thymohydroquinone have been reported to have an antibacterial activity and beneficial interaction with some antibiotics [14]. In Ethiopia black cumin seed and oil are used in folk medicine, as a bread flavoring and as a spices. The aim of this study is to describe the chemical composition of essential oil black cumin seeds cultivated in Ethiopia.

\section{MATERIALS AND METHODS}

2.1. Isolation of essential oils ;

The collected seeds were air dried, ground with a laboratory mill, weighed and particular samples (100g) from each varieties were subsequently submitted to hydro-distillation for $4 \mathrm{~h}$ using Clevenger type apparatus. Three replicates were distilled simultaneously for each. All oil samples were weighed, dried over anhydrous sodium sulphat and stored in dark at $4^{\circ} \mathrm{C}$ to identification of chemical constituent. The yields obtained were averaged and calculated as a relative percentage $(\mathrm{V} / \mathrm{W})$.

\subsection{Gas chromatography $(G C)$}


GC analyses were performed by using Agilent 7890B gas chromatography equipped with a DB5 fused silica column $(30 \mathrm{~m} \times 0.25 \mathrm{~mm}$ i.d., film thickness $0.25 \mu \mathrm{m})$. Oven temperature was held at $40^{\circ} \mathrm{Cfor} 5 \mathrm{~min}$ and then programmed until $250^{\circ} \mathrm{C}$ at a rate of $4^{\circ} \mathrm{C} / \mathrm{min}$. Injection and detector (FID) temperature were $260^{\circ} \mathrm{C}$; Helium was used as carrier gas.

\subsection{Gas chromatography- Mass spectrometry ( $G C$-MS $)$}

GC-MS analyses were carried out Agilent 5977A system equipped with a DB-5MS fused silica column (30 m x $0.25 \mathrm{~mm}$; film thickness $0.25 \mu \mathrm{m}$ i.d.,). Oven temperature was 40 to $240^{\circ} \mathrm{C}$ at arate of $4^{\circ} \mathrm{C} / \mathrm{min}$, transfer line temperature $260^{\circ} \mathrm{C}$, injector temperature $250^{\circ} \mathrm{C}$, carrier gas Helium was used split ratiol/60, flow rat $1 \mathrm{ml} / \mathrm{min}$. Ionization energy $70 \mathrm{ev}$, scan time 1s; mass rang 40-350 amu.

\subsection{Identification of compounds}

The constituent of the oils were identified by comparison of their mass-spectra with those of a computer NIST 2014. library and confirmed by comparison of their compounds. Adams, (1995). [15]. Retention indices were determined using retention times of n-al-kanes that have been injected to the same instrument and under the same chromatographic condition. Relative percentage amounts were calculated from the total area under the peak by the software of the apparatus.

\section{Results and Discussions}

Hydro-distillation of Ethiopian black cumin seeds were yellowish oil and resulted in $0.80 \%, 0.60 \%$ and $0.40 \%$ essential oil for Eden, Dershay and Silengo varieties respectively. The obtained result in this study was confirms that the variation in essential oil content with in variety and comparable with many authors report. Prabudha and Ria, [16] reported that the percentage of volatile oils of cumin seeds were ranged from 0.5 to $1.5 \%$. Kokoska L. et al., (2008) [18] reported that the cumin seeds essential oil was $0.29 \%$.

Table 1; Chemical composition of black cumin seeds verities Essential oil

No Identified compounds

P- Cymene

$\alpha$-Thujane

trans-4-methoxy thujane

9,12-Octadecanoic acid, (Z,Z)-

$\beta$-pinene

$\alpha$ - pinene

gamma-terpinene

cis-vaccenic acid

Thymoquinone

Longifolene

D-limonene

Phenol-2-methyl-5-(1-methylethyl)-

Bicyclo[3,1,0]hexane,4-methylene-1-(1-methylethyl)

Terpinen-4-ol

cis-4-methoxy thujane

n-hexadecanoic acid

1,3-Cyclohexadiene, 1-methyl-4-(1-methylethyl)

1-Cyclohexene-1-carboxaldehyde, 2,6,6-trimethyl

Behenic alcohol

9,12-octadecadieenoicacid

(hydroxymethyl)ethylester

21 2-caren-4-ol

22 Tricyclo[5,4,0,0(2,8)undec-9-ene, 2,6,6,9-

tetramethyl-, (1R,2S,7R,8R)

Glycidyl oleate

Bicycle[2,2,1]heptan-2-ol,1,7,7-trimethyl-,acetate,endo

p-Mentha-1,5,8-triene

Oleic acid

Isoledene

7-Tetradecenal, $(Z)$

Glycidyl palmitate variety

Eden (\%) Dershy Selingo

$\begin{array}{lll}7.39 & 7.91 & 8.86\end{array}$

$\begin{array}{lll}6.04 & 0.07 & 0.16\end{array}$

$\begin{array}{lll}3.04 & 4.08 & 3.45\end{array}$

$\begin{array}{lll}2.68 & 3.94 & 3.00\end{array}$

$\begin{array}{lll}2.50 & 3.83 & 3.24\end{array}$

2.46

2.13

1.83

2.08

1.60

1.52

1.13

1.06

0.96

0.93

0.91

0.88

(Z,Z)-,2-hydroxy-1-

0.61

0.34

0.30

0.43

$2.40 \quad 3.53$

$1.56 \quad 1.95$

$2.29 \quad 2.27$

$1.18 \quad 2.56$

$2.29 \quad 1.87$

$0.92 \quad 1.31$

$1.30 \quad 1.35$

$0.02 \quad 0.04$

$0.93 \quad 1.00$

$0.13 \quad 0.40$

$0.03 \quad 0.02$

0.22

$0.02-$

$\begin{array}{lll}0.18 & 0.16 & 0.26\end{array}$

$\begin{array}{lll}0.15 & - & 0.10\end{array}$

$0.13 \quad 0.03 \quad 0.06$

$\begin{array}{lll}0.12 & 0.1 & 0.12\end{array}$

$\begin{array}{lll}0.12 & 0.08 & 0.13\end{array}$

0.10 


\begin{tabular}{|c|c|c|c|c|}
\hline \multirow[t]{2}{*}{ No } & \multirow[t]{2}{*}{ Identified compounds } & \multicolumn{3}{|l|}{ variety } \\
\hline & & Eden $(\%)$ & $\begin{array}{l}\text { Dershy } \\
(\%)\end{array}$ & $\begin{array}{l}\text { Selingo } \\
(\%)\end{array}$ \\
\hline 30 & Cyclohexanol, 1-methyl-4-(1-methylethenyl)-,cis- & 0.10 & & 0.10 \\
\hline 31 & Benzenemethanol, $\alpha, \alpha, 4$-trimethyl & 0.10 & 0.04 & 0.06 \\
\hline 32 & $(-)$-Carvone & 0.09 & 0.08 & 0.15 \\
\hline 33 & Bicycle[3,1,0]hexan-2-ol,2-methyl-5-(1-methyethyl)-(1 $\alpha, 2 \beta, 5 \alpha)$ & 0.08 & 0.07 & 0.08 \\
\hline 34 & Cyclohexene,1-methyl-4-(methylethyl) & 0.08 & 0.09 & 0.08 \\
\hline 35 & Eucalyptol & 0.07 & 0.08 & 0.07 \\
\hline 36 & $\begin{array}{l}\text { 1,2,4-methenozulene, decahydro- } \\
\left.\left(1 \alpha, 2 \alpha, 3 \alpha \beta, 4 \alpha, 8 \alpha \beta, 9 \mathrm{R}^{*}\right)\right]\end{array}$ & 0.07 & & 0.07 \\
\hline 37 & Caryophylene & 0.07 & 0.06 & 0.08 \\
\hline 38 & Humulane-1,6-dien-3-ol & 0.07 & 0.04 & 0.07 \\
\hline 39 & p-Cymenene & 0.06 & 0.06 & 0.08 \\
\hline 40 & 1,2-15,16-diepoxyhexadecane & 0.06 & & \\
\hline 41 & $\begin{array}{l}\text { (1R,3R,4R,5S)-1-Isopropyl-4-methylbicyclo[3,1,0]hexan-3-yl } \\
\text { acetat-rel- }\end{array}$ & 0.05 & 0.04 & 0.06 \\
\hline 42 & Tetradecanal & 0.06 & 0.04 & 0.08 \\
\hline 43 & $\alpha$-Terpineol & 0.04 & 0.02 & 0.04 \\
\hline 44 & Camphene & 0.04 & 0.06 & 0.05 \\
\hline 45 & $\alpha$-phellandrene & 0.04 & 0.06 & 0.06 \\
\hline 46 & 2-Cyclohexen-1-ol,1-methyl-4-(methylethyl)-,cis & 0.04 & 0.02 & 0.04 \\
\hline 47 & 1H-Indene, 1,ethylideneoctahydro-7a-methyl-, cis- & 0.04 & 0.04 & 0.07 \\
\hline 48 & Benzene, 1-(1,5-dimethyl-4-hexenyl)4-methyl & 0.04 & 0.04 & 0.06 \\
\hline 49 & Ar-tumerone & 0.04 & 0.04 & 0.10 \\
\hline 50 & $\begin{array}{l}\text { Phenanthrene, 7-ethyl-1,2,3,4,4a,4b,5,6,7,9,10,10a-dodecahydro- } \\
\text { 1,1,4a,7-tetramethyl-,[4aS- }(4 \mathrm{a} \alpha, 4 \mathrm{~b} \beta, 1 \beta, 10 \mathrm{a} \beta)]\end{array}$ & 0.04 & 0.02 & 0.04 \\
\hline 51 & Tetradecanoic acid, 2-hydroxy & 0.04 & - & - \\
\hline 52 & Bicycle[3,1,0]hex-2-ene,4-methylene-1-(1-methylethyl) & 0.02 & 0.03 & 0.03 \\
\hline 53 & $\beta$-myrcene & 0.03 & 0.04 & 0.04 \\
\hline 54 & Thymol & 0.03 & 0.02 & 0.04 \\
\hline 55 & Cis-9-tetradecen-1-ol & 0.03 & 0.02 & 0.07 \\
\hline 56 & 2-Caren-4-ol & 0.02 & 0.02 & \\
\hline 57 & 1,3,8-p-Menthatriene & 0.02 & 0.02 & 0.02 \\
\hline 58 & $(+)-($ E)-Limonene oxide & 0.02 & 0.02 & 0.03 \\
\hline 59 & $\beta$-Bisabolene & 0.02 & 0.02 & 0.03 \\
\hline 60 & trans-2-Decen-1-ol,trifluroacetate & 0.02 & - & - \\
\hline 61 & Dodecanal & 0.02 & 0.01 & 0.04 \\
\hline 62 & p-Cymene-2,5-diol & 0.02 & 0.04 & 0.06 \\
\hline 63 & Tumerone & 0.02 & 0.02 & 0.04 \\
\hline 64 & Curlone & 0.02 & 0.02 & 0.04 \\
\hline 65 & 7-Hexadecenal,(Z) & 0.02 & - & - \\
\hline 66 & 1,5-Dodecadiene & 0.01 & - & - \\
\hline 67 & $\alpha$-Terpinene & - & 1.26 & 3.00 \\
\hline 68 & 7-Oxabicyclo[4,1,0]heptane, 1-methyl-4-(2-methyloxiranyl) & - & 0.01 & 0.02 \\
\hline 69 & endo-Borneol & - & 0.03 & 0.05 \\
\hline 70 & 2-cyclohexen-1-ol, 1-mrthyl-4-(1-methylethyl)-,trans & - & 0.02 & 0.02 \\
\hline 71 & $\alpha$-Copaene & - & 0.02 & - \\
\hline 72 & Citronellal & - & - & 0.08 \\
\hline 73 & 1,2,5,9-Tetradecatriene,3,12-diethyl & - & - & 0.09 \\
\hline 74 & Cyclohexene, 1-methyl-4-(1-methylethylidene) & - & - & 0.09 \\
\hline 75 & E-7-Tetradecenol & - & - & 0.08 \\
\hline 76 & Bicycle[2,2,1]heptane-2-one, 1,7,7-trimethyl-,(1S) & - & - & 0.04 \\
\hline 77 & Benzaldehyde,4-(methylethyl) & - & - & 0.02 \\
\hline 78 & Carvenone & - & - & 0.01 \\
\hline 79 & 2-cyclohexen-1-one, 3-methyl-6-(methylethylidene) & - & - & 0.02 \\
\hline 80 & $\alpha$-Cubebene & - & - & 0.02 \\
\hline 81 & 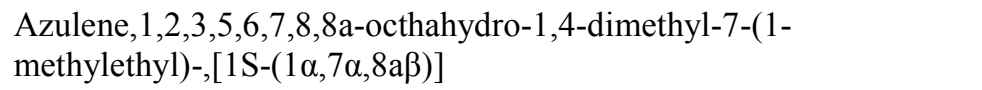 & - & - & 0.03 \\
\hline
\end{tabular}




\section{No Identified compounds}

82

83

84

85

Total

1-Dodecanol

2-ene tetramethyl

variety

Eden (\%) Dershy Selingo

$(\%) \quad(\%)$

0.04

0.02

(1S,5S)-2-Methyl-5-(R)-6-methylhept-5-en-2-yl)bicyclo[3,1.0]hex-

1H-benzocyclohepten-9-ol,2,4a $\beta, 5,6,7,8,9,9 \mathrm{a} \beta$-octahydro-3,5,5,9 $\beta$ -

Data in table 1 showed identified black cumin seeds essential oils constituents were analyzed using GC-MS. From the GC-MS result sixty six, sixty and seventy five constituents were identified which constituting $99.8 \%$, $99.27 \%$ and $99.17 \%$ in Eden, Dershay and Selingo black cumin seeds essential oils. The main constituents of black cumin seeds EO as detected by GC/MS were p-cymene (44.36\%), $\alpha$-thujen (12.57\%), trans-4-methoxy thujane (7.39), 9,12-Octadecanoic acid, 9Z,Z)- (6.04\%), $\beta$-pinene (3.04\%), $\alpha$-pinene (2.68), gamma-terpinene $(2.50 \%)$, cis-vaccenic acid $(2.46 \%)$, thymoquinone $(2.13 \%)$, D-limonene $(2.08 \%)$, longifolene $(1.83 \%)$, phenol2-methyl-5-(1-methylethyl) (1.60\%), bicyclo[3,1,0]hexane,4-methylene-1-(1-methylethyl) $(1.52 \%)$, terpinen-4-ol $(1.13 \%)$, cis -4-methoxy thujane $(1.06 \%)$ in eden variety, p-cymene (45.85\%), $\alpha$-thujen $(17.30 \%)$, trans-4-methoxy thujane (7.91), $\beta$-pinene (4.08\%), $\alpha$ - pinene (3.94), gamma-terpinene (3.83\%), thymoquinone (2.40\%), D-limonene $(2.29 \%)$, longifolene $(1.56 \%)$, bicyclo[3,1,0]hexane,4-methylene-1-(1-methylethyl) $(1.52 \%)$, cis -4-methoxy thujane $(1.30 \%)$, $\alpha$-terpinene $(1.26 \%)$, phenol-2-methyl-5-(1-methylethyl) $(1.18 \%)$ in dershay variety and and pcymene $(44.31 \%), \alpha$-thujen $(13.66 \%)$, trans-4-methoxy thujane $(8.86 \%)$, thymoquinone $(3.53 \%)$, $\beta$-pinene $(3.45 \%)$, gamma-terpinene (3.24\%), $\alpha$ - pinene (3.00), $\alpha$-Terpinene (3.00), \%), phenol-2-methyl-5-(1-methylethyl) $(2.56 \%)$, D-limonene $(2.27 \%)$, bicyclo[3,1,0]hexane,4-methylene-1-(1-methylethyl) $(1.87 \%)$, longifolene $(1.56 \%)$, 1-Cyclohexene-1-carboxaldehyde (1.53), 2,6,6-trimethyl terpinen-4-ol (1.31\%), cis -4-methoxy thujane $(1.35 \%)$ in variety. The p-cymene and $\alpha$-Thujane, $\beta$-pinene, $\alpha$ - pinene, gamma-terpinene, Bicyclo[3,1,0]hexane,4methylene-1-(1-methylethyl) content of dershy. From Iran black cumin seeds essential oil 32 compounds were identified and the main compounds of the essential oil were trans anethole (38.3\%), p-cymene (14.8\%), limonene $(4.3 \%)$, and carvone (4.0\%) Nickavar B. et al., (2003) [17]. The present study revealed that the essential oil isolated from each black cumin variety seeds cultivated in ethiopia are similar to each other and the others country black cumin essential oil in respect of presence of main compounds. Bahman. N. et al., (2003) [17], Kokoska.L. et al., (2008) [18], Khalid.A.K. et al., (2016) [19].

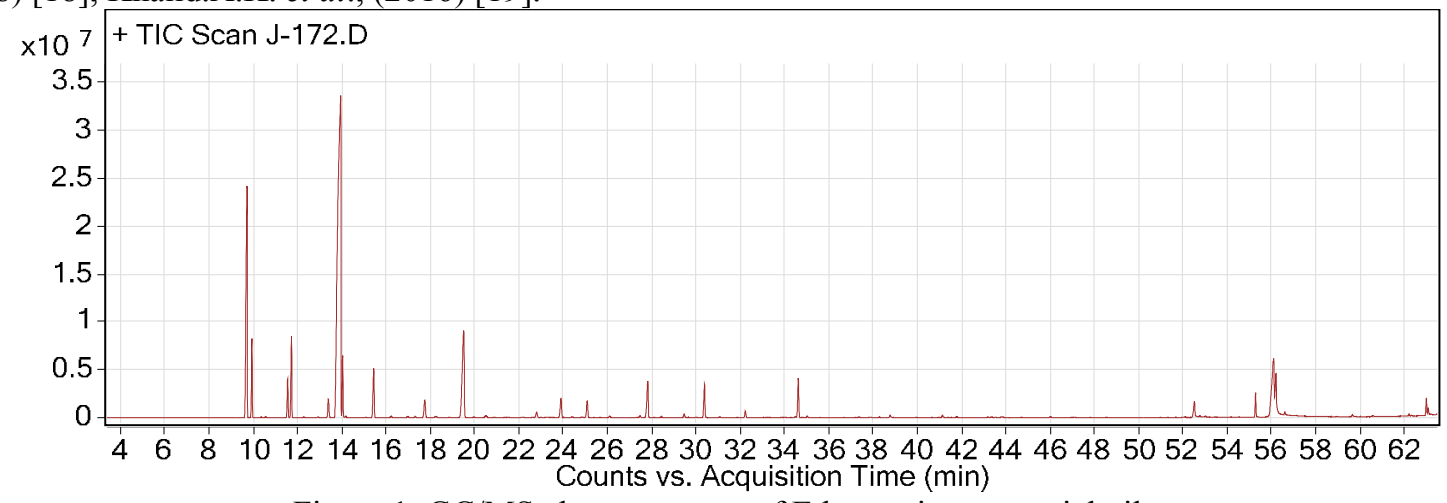

Figure 1. GC/MS chromatogram of Eden variety essential oil

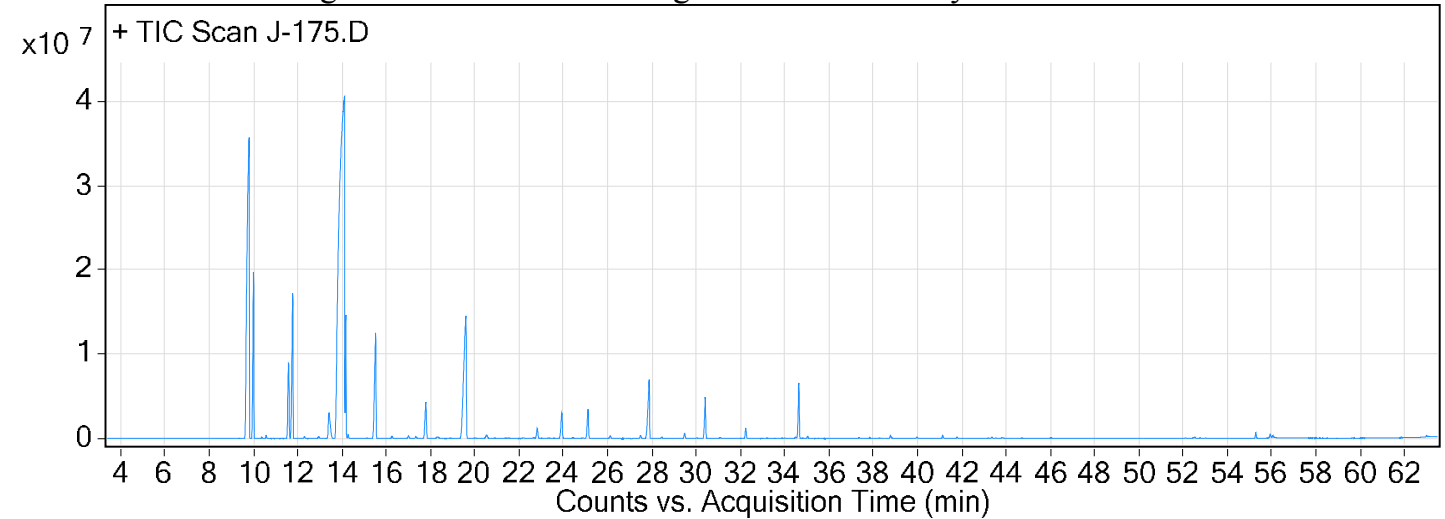

Figure 2. GC/MS chromatogram of Dershaye variety essential oil 


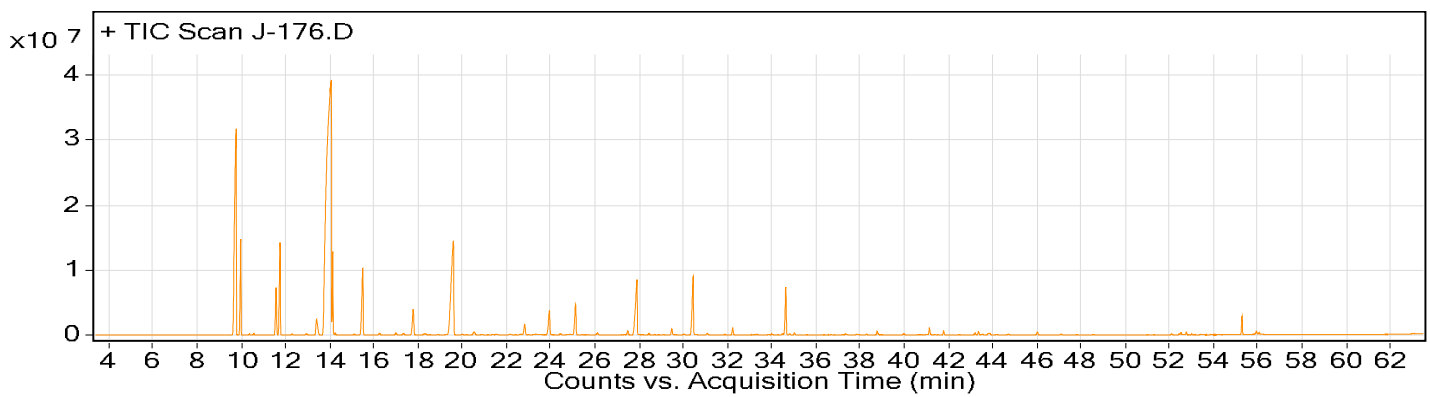

Figure 3. GC/MS chromatogram of Selingo variety essential oil

\section{Conclusion}

Essential oil content and composition of black cumin affected by types of genetic material, ecological condition and origin of the plant. The result obtained from the study indicates significant variations on black cumin essential oil and chemical composition content from three varieties cultivated in the same agro-ecology. p-cymene (45.85 $44.31 \%), \alpha$-thujen $(17.30-12.57 \%)$, trans-4-methoxy thujane (8.86 - 7.39\%), 9,12-Octadecanoic acid, 9Z,Z)$(6.04 \%-0.07 \%), \quad \beta$-pinene $(4.08-3.04 \%)$, $\alpha$ - pinene $(3.94-2.68 \%)$, gamma-terpinene $(3.83-2.50 \%)$, thymoquinone $(3.53-2.13 \%), \alpha$-terpinene $(3.00-0.00 \%)$, D-limonene $(2.29-2.08 \%)$, phenol-2-methyl-5-(1methylethyl) (2.56-1.52\%), cis-vaccenic acid (2.46 - 0.00\%), longifolene (1.95 - 1.83\%), terpinen-4-ol (0.92 $1.31 \%)$ and cis-4-methoxy thujane $(1.35-1.06 \%)$ are the major constituents of the three varieties of black cumin essential oils cultivated in Ethiopia. The Ethiopian black cumin essential oil contain the required major secondary metabolite for pharmacological and other application.

\section{Acknowledgment}

The authors would to thank Ethiopian Institute of Agricultural Research (EIAR) for their financial support of this work.

\section{Conflict of interest}

The authors would like to declare that the study was carried out mainly for academic research purpose without any conflict of interest.

\section{Reference}

1. Farrell KT. (1990). Spices, Condiments, and Seasoning. $2^{\text {nd }}$ New York ;Van Nostrand Renhold.

2. Walker JRL.(1994). Antimicrobial compounds in food plants. In Dillon VM, Road RG, eds. Natural antimicrobial systems and food preservation. Wallingford (UK): CAB International. 181-204.

3. Srinivasan K. (2005). Role of spices beyond food flavouring : nutraceuticals with multiple health effects. Food Rev. In; 21: 167-188.

4. Hossain MB, Bruntan NP, Barry-Ryan C, Martin-Diana, AB, Wilkinson M. (2008). Antioxidant activity of spices extracts and phenolics comparison of synthetic antioxidants. Rasayan J. Chem. 1:751-756.

5. Wais A., Radoslaw Bonikowski and Danuta Kalemba ( 2008). Composition of essential oil from seeds of Nigella sativa L cultivated in Poland. Flavor and Fragrance 23;126-132.

6. Ali B.H, and G. Blunden. (2003). Pharmacological and toxicological properties of Nigella sativa.L. Phytother,Res.17:299-305.

7. Abdulelah HAA, Zainal-Abidin BAH. (2007). In Vivo Antimalarial test of Nigella sativa . (Black Seed) Different Extracts. AMJ.Pharmacol Toxicol.2:46-50.

8. Buyukozturk G, Gelincik A, Ozaseker F, Genc S, Savran FO, Kiran B, et al., (2005). Nigella sativa (Black seed) oil does not affect the T-helper 1 and $\mathrm{T}$ - helper 2 type cytokine production from splenic mononuclear cells in allergen sensitized mice. J Ethnopharmacol. 100:295-298.

9. Majdalawieh AF, Hmaidan RI, Carr RI. (2010). Nigella sativa modulates splenocyte proliferation, Th1/Th2 cytokine profile, macrophage function and NK anti-tumor activity. J Ethopharmacol131:268-275.

10. Salem ML, Hossain MS. (2010). Protective effect of black seed oil from Nigella sativa against murine cytomegalovirus infection. Int J Jmmunopharmacol. 22:792-740.

11. Kamal A, Arif JM, Ahmad IZ. (2010). Potential of Nigella sativa L. seed during different phases of germination on inhibition of bacterial growth.E3 J Biotechnol pharmaceut Res. 1:9-13.

12. Meddah B, Ducroc R, Faouzi ME, Mahraoui L, Benhaddou-Andaloussi A, Martineau LC et al. (2009).Nigella sativa inhibits intestinal glucose absorption and improves glucose tolerance in rats. J Ethnopharmacol. 121:419-429.

13. Oktay AS, Ethem GT, Omer FA. (2005). The protective effect of thymoquinone on ethanol-induced acute 
gastric damage in the rat. Nutr Res. 25:673-680.

14. Halawani E. (2009). Antibacterial Activity of thymoquinone and thymohydroquinone of Nigella sativa L. and Their Interaction with Some Antibiotics. Adv Biol Res. 3:5-6, 148-152.

15. Adams R P, (1995). Identification of essential oil components by gas chromatography/mass spectrometry. Allured Carol Stream, lllinois, USA.

16. Prabudha A, and Rai T,. (2002). Herbs and spices - potential dairy ingridents. The Beverage and Food World. 85(8):35-47

17. Nickavara B, F Mojaba, K Javidniab and M A R Amoli. (2003).Chemical Composition of the Fixed and Volatile Oils of Nigella sativa L. from Iran. Z.Naturforsch. 58c:629-631.

18. Kokoska L, Havlik J, Valterova I, Sovova H, Sajfrtova M, and Jankovska L. (2008). Comparison of chemical composition and antibacterial Activity of Nigella sativa seed Essential Oils obtained by Different Extraction Methods. J Food protection. 17(12):2475-2480.

19. Khalid A K, and Shedeed M R. (2016). GC-MS analyses of black cumin essential oil produces with sodium chloride. Int. Food Ras J. 23(2):832-836. 